\title{
qSOFA should replace SIRS as the screening tool for sepsis
}

\author{
Stefano Franchini ${ }^{*}$ and Andrea Duca
}

See related research by Vincent et al., https://ccforum.biomedcentral.com/articles/10.1186/s13054-016-1389-z

Vincent JL, Martin GS and Levy MM recently wrote an article in Critical Care entitled "qSOFA does not replace SIRS in the definition of sepsis" [1]. In this paper they specified that "the qSOFA is meant to be used to raise suspicion of sepsis and prompt further action but it is not a replacement for SIRS and is not part of the definition of sepsis".

One of the starting points that induced the Sepsis-3 consensus taskforce to set out in search of better entry criteria than the systemic inflammatory response syndrome (SIRS) criteria was precisely that SIRS criteria perform poorly on both "discriminant validity" and "convergent validity"[2]. In order to accomplish their task, they identified patients with suspected infection among 1.3 million health record cases and, after comparing the performance of several different clinical criteria, they came out with the quick sequential organ failure assessment (qSOFA) score, whose predictive validity for inhospital mortality outside the ICU was statistically better than SIRS [3].

The fact that nonspecific SIRS criteria will "generally" continue to aid in the identification and diagnosis of infection was repeatedly affirmed in the Sepsis-3 consensus article [2]. Besides, when the
SIRS criteria were first proposed as a screening tool for sepsis [4], they were meant to be applied to patients with "suspected infection", just as the qSOFA is intended to be used now. However, while the SIRS criteria were essentially based only on expertconsensus [4], the qSOFA criteria were identified through large multivariate statistics and confirmatory analyses, where they proved to perform better than the SIRS criteria [3].

The qSOFA was derived and conceived on the basis of retrospective data, and thus, from now on, the clinical research should and will work hard to prospectively validate the soundness of this tool, in terms of its screening capacity. However, based at least on the currently available evidence, we believe that, although qSOFA does not replace SIRS in the definition of sepsis, it should indeed replace SIRS as the screening tool for sepsis.

We would like to know if Vincent and colleagues agree with this assumption, and we would also like to ask them if, after the Sepsis-3 consensus definitions, the SIRS criteria still retain a real operative role in the process of defining and/or screening sepsis or if they could be, at least operatively, dismissed.

\section{Authors' response}

Jean-Louis Vincent, Greg S. Martin and Mitchell M. Levy

We thank Drs Franchini and Duca for their comments. Physicians have long used fever, associated tachycardia and altered white blood cell count as signs of infection ... we have never needed the SIRS criteria to help with this and we don't need the qSOFA for this either. Furthermore, qSOFA does not replace SIRS as a

\footnotetext{
* Correspondence: franchini.stefano@hsr.it

Emergency Department, Ospedale San Raffaele Scientific Institute, Via Olgettina 60, Milan 20132, Italy
}

screening tool for sepsis because it was conceived, derived and validated as a prognostic tool. Moreover, sepsis is more often identified from associated unexplained organ dysfunction than from infection [5].

The use of qSOFA as an alarm signal should be further validated, keeping in mind that it is not specific for sepsis. Patients with many other conditions, including severe heart failure, blood loss, pulmonary embolism and any form of acute circulatory failure (shock), can have hypotension, altered mental status and hyperventilation, 
thus meeting the qSOFA criteria without having sepsis. But, it is still important to identify these patients and act quickly, whatever the underlying cause. The best screening tools for sepsis remain within the minds of clinicians, suspecting infection and assessing organ function using an array of criteria that so far have eluded complete description.

\section{Abbreviations}

qSOFA: quick sequential organ failure assessment; SIRS: systemic inflammatory response syndrome

\section{Acknowledgements}

None.

\section{Funding}

The authors certify that no funding has been received for the conception and writing of this article.

\section{Availability of data and materials}

Not applicable.

\section{Authors' contributions}

SF was responsible for conception of the letter, and drafted and revised the manuscript. AD conceived the letter and helped to draft the manuscript. All authors read and approved the final manuscript.

\section{Authors' information}

Both SF and AD are emergency physicians working full-time in the Emergency Department and are involved in their institution's teaching program regarding sepsis for physicians and nurses.

\section{Competing interests}

The authors declare that they have no competing interest.

\section{Consent for publication}

Not applicable.

\section{Ethical approval and consent to participate}

Not applicable.

Received: 6 September 2016 Accepted: 8 November 2016

Published online: 28 December 2016

\section{References}

1. Vincent JL, Martin GS, Levy MM. GSOFA does not replace SIRS in the definition of sepsis. Crit Care. 2016;20:210.

2. Singer M, Deutschman CS, Seymour CW, Shankar-Hari M, Annane D, Bauer M, et al. The Third International Consensus Definitions for Sepsis and Septic Shock (Sepsis-3). JAMA. 2016;315:801-10.

3. Seymour CW, Liu VX, Iwashyna TJ, Brunkhorst FM, Rea TD, Scherag A, et al. Assessment of Clinical Criteria for Sepsis: For the Third International Consensus Definitions for Sepsis and Septic Shock (Sepsis-3). JAMA. 2016;315:762-74.

4. Bone RC, Balk RA, Cerra FB, Dellinger RP, Fein AM, Knaus WA, et al. Definitions for sepsis and organ failure and guidelines for the use of innovative therapies in sepsis. The ACCP/SCCM Consensus Conference Committee. American College of Chest Physicians/Society of Critical Care Medicine. Chest. 1992;101:1644-55.

5. Vincent JL, Mira JP, Antonelli M. Sepsis: older and newer concepts. Lancet Respir Med. 2016:4:237-40. 